\title{
Involvement of Ischemia-driven 5LPOX-RvE1- ChemR23 axis in the resolution of post-CABG inflammation in Coronary Arteries
}

Finosh Thankam ( $\nabla$ fthankam@westernu.edu )

Western University of Health Sciences https://orcid.org/0000-0001-7285-9808

Victoria E. D. Wilson

Queen's University Belfast

Mohamed M Radwan

Western University of Health Sciences

Aleem Siddique

University of Nebraska Medical Center

Devendra K Agrawal

Western University of Health Sciences https://orcid.org/0000-0001-5445-0013

\section{Research Article}

Keywords: Coronary artery bypass grafting, Ischemia, Ischemic heart diseases, Lipoxygenases, Proresolvin lipid mediators, Reperfusion

Posted Date: November 17th, 2021

DOI: https://doi.org/10.21203/rs.3.rs-1064912/v1

License: (c) (i) This work is licensed under a Creative Commons Attribution 4.0 International License. Read Full License 


\section{Abstract}

Aims: Expression status of pro-resolving lipid mediators (PLM) and receptors in the post-CABG coronary arteries are largely unknown. Here, we aim to investigate the expression of PLMs in the atherosclerotic post-CABG swine LAD compared to without CABG (LAD-AS), and in isolated coronary artery smooth muscle cells (CASMCs) cultured under ischemia.

Methodology: The arteries of interest were harvested from post-CABG atherosclerotic swine and the histomorphology and the expression status of key PLM mediators were quantified using immunostaining. SMCs were cultured under ischemia and confirmed the expression on PLM mediators at transcript and protein level.

Results: The histomorphometric analysis revealed considerable alterations in the tissue architecture in LAD-CABG and LAD-AS arteries compared to control. PLM synthetic enzyme 5LPOX was significantly upregulated in LAD-CABG and LAD-AS whereas the other mediators including 12LPOX, 15LPOX, COX2, ChemR23, GPCR18, GPCR120 were decreased in LAD-CABG than control. LPOX enzymes and PLM receptors were upregulated in ischemic CASMCs with respect to control. Western blot showed the upregulation of 5LPOX, and ChemR23. Additionally, higher level of RvE1 was observed in ischemic control CASMCs which was decreased following reperfusion.

Conclusion: These findings suggest that the CASMCs withstand the ischemia-triggered proinflammatory episodes by increasing the secretion of RvE1 mediated through 5LPOX and ChemR23 signaling.

\section{Introduction}

Hyperactivation of inflammatory events and delayed/impaired resolution of inflammation exacerbates cardiovascular disease (CVD) pathology [1]. The increased pro-inflammatory episodes, especially in coronary atherosclerotic events resulting in ischemia and reperfusion, remain consistent with the impaired resolution of inflammation, which aggravates the pathology of ischemic heart diseases (IHD) [1]. Similarly, the sustained ischemic events impede the resolution of inflammation and prevent the functional recovery of myocardium following an infarction [2]. A basic understanding regarding the mechanism underlying the resolution of chronic unresolved inflammation in atherosclerosis and IHD is limited; however, it possesses immense translational potential. Interestingly, versatile classes of polyunsaturated fatty acids (PUFAs)-derived pro-resolvin lipid mediators (PLMs) have been identified, notably the $E$ and $D$ series of resolvins, protectins, maresins, and lipoxins [3]. The biochemical transition of docosahexanoids and eicosanoids activate the expression, localization, and activities of cellular lipoxygenases (LPOX) including 5-, 12- and 15-LPOX deviating from their conventional synthetic pathways of proinflammatory prostaglandins and leukotrienes [4]. The three LPOX family enzymes play key roles in the synthesis of PLMs, which in turn mediate the crosstalk between inflammatory cells and local cell phenotypes and promote the resolution of inflammation mainly via a promotion of the transition of M1-M2 macrophages [5]. 
Generally, PLM facilitate the resolution of inflammation by upregulating specific LPOX activities along with the expression and presentation of PLM receptors on the surface of leukocytes and host cells [3] [6]. However, the PLM signaling in vascular cells (including SMCs and endothelial cells) under pathological simulations remains unknown. Generally, the PLM synthesis pathway utilizes PUFAs, including eicosapentaenoic acid (EPA) and docosahexaenoic acid (DHA) via cyclooxygenase (COX)2 and LPOX activities, and leads to the formation of RvD and RvE family resolvins and maresins [7] [6]. Interestingly, the treatment with PLM accelerated the resolution of inflammatory signaling in IHD, as evident by the accelerated resolution of inflammation and healing response following myocardial infarction (MI) in human patients and animal models [8], [9].

While coronary artery bypass graft (CABG) surgery is the established lifesaving strategy for IHD, an increased pro-inflammatory pool has been reported in the vein graft and associated blood vessels in versatile CABG models [10], [11]. However, the expression status of PLM receptors and mediators, as well as downstream signaling has yet to be documented. A clearer understanding of PLM induction signaling would reveal the underlying molecular mechanisms and open novel translational avenues. Moreover, ischemic insults and inflammation persist in the post-CABG coronary artery due to the increased level of oxidative stress and damage associated molecular patterns (DAMPs) [10]. However, the expression status of PLM mediators and receptors in the post-CABG coronary arteries is largely unknown. The impact of ischemic and reperfusion events on PLM mediators in coronary artery smooth muscle cells (CASMCs) remain unexplored. Considering the unknowns, the present study aimed to investigate the expression of PLM mediators, including the key enzymes COX2, 5LPOX, 12LPOX, and 15LPOX, as well as the major receptors, including 7- transmembrane G-protein coupled receptor (GPCR)18, GPCR120 and chemokine like receptor 1 (ChemR23) in the left anterior descending (LAD) coronary artery of post-CABG swine model and isolated coronary SMCs.

\section{Materials And Methods}

\section{Animals and experimental groups}

The Institutional Animal Care and Use Committees of Creighton University, Omaha, Nebraska and Western University of Health Sciences, Pomona, California, approved the study. Animal management functioned strictly in accordance with the $\mathrm{NIH}$ and OLAW guidelines throughout the study and extreme care was taken to minimize distress to the animals. The study involved three experimental groups $(n=3$ animals in each group): Group I (LAD-CABG) - atherosclerotic Yucatan microswine (Sus scorfa, Sinclair bioresources) which underwent CABG surgery, Group II (LAD-AS) - atherosclerotic Yucatan microswine without CABG surgery, and Group III (LAD-C) - normal miniswine (Sus scorfa, SBS farms). Microswine (female, 8-10 months of age, weighing 60-80 pound) and miniswine (female, 6-8 months, 50-60 pounds) were acclimatized for one week, and maintained in a 12/12 light-dark cycle. The microswine were fed a high-cholesterol and high-fat diet, whereas the miniswine were fed with a normal diet throughout the study. The animals maintained uninterrupted access to water throughout the study. 


\section{CABG surgery and LAD harvest}

The CABG procedure was performed in LAD-CABG group by grafting the superficial epigastric vein (SEV) between the left internal mammary artery (LIMA) and the left anterior descending (LAD) artery per the procedure described in our recent publication [10]. Surgical procedures typically simulated human CABG. The animals continued to be fed with a high-cholesterol and high-fat diet post-CABG and euthanized 6 months post-procedure to harvest the LAD distal to the anastomoses for analysis. Similarly, the LAD was harvested from the animals of LAD-AS and LAD-C groups from an identical anatomical location. The harvested tissues were processed for formalin fixation and paraffin embedding for histology and immunofluorescence analysis.

\section{Histology}

The sections ( $5 \mu \mathrm{m}$ thick) were deparaffinized and $\mathrm{H} \& \mathrm{E}$, Trichrome, and pentachrome staining were performed to examine the tissue morphometry, ECM composition, and architecture following our previously reported protocol [12], [13]. Stained tissues were mounted using xylene-based mounting media and imaged using a fluorescent slide scanner system (Thunder, Leica) at 20x magnification. The images were processed using LASX software (Leica) and saved in JPEG format.

\section{Immunofluorescence}

Protein expression of key biomarkers involved in resolvin biology from the harvested LAD tissue sections was analyzed by immunofluorescence following our previously reported protocol [14], [12]. The deparaffinized sections were briefly subjected to antigen retrieval in HIER buffer (Heat Induced Antigen Retrieval) (TA-135-HBM) at $95^{\circ} \mathrm{C}$ for 20 min and blocking ( $0.25 \%$ Triton $\mathrm{X}-100$ and $5 \%$ horse serum in PBS) at room temperature for $90 \mathrm{~min}$. Sections were then incubated overnight at $4^{\circ} \mathrm{C}$ with the primary antibodies (1:300 dilution) against the enzymes 5LPOX (ab-39347), 12LPOX (ab-211506), 15LPOX (ab244205), and COX2 (ab-23672), as well as the receptors ChemR23 (ab-230442), GPCR18 (ab-76258), and GPCR120 (ab-230869). The fluorochrome-conjugated secondary antibodies (1:400) were used to detect the primary antibodies. Nuclei were counterstained with 4',6-diamidino-2-phenylindole (DAPI) (H-1200) and imaged using the fluorescent slide scanner system (Thunder, Leica) at 20x magnification and the images were acquired using LASX software (Leica). The raw images were processed for background clearance and cell counting and mean fluorescence intensity (MFI) corresponding to the expression of the proteins was quantified using $\mathrm{NIH}-$-ImageJ software and normalized to 100 cells (MFI/100 cells). Three-tofour images were randomly acquired from each specimen to quantify MFI/100 cells and the average value was utilized for statistical analysis. From MFI/100 cells the variation with respect to the control (VRC) (ratio of respective MFI/100 cells of the specimen to that of average control) was calculated and the protein expression was represented as fold-change expression calculated by transforming VRC to 
logarithmic $\left(\log _{2}\right)$ scale. A negative control was employed to fix the exposure time and to clear the background.

\section{SMC isolation, culture and simulation of ischemia and reperfusion}

Coronary artery SMCs (CASMCs) were isolated from the LAD of the microswine with the use of the collagenase digestion method and characterized following our previously established protocol [10] [15]. The cells were maintained in SMC growth media containing 10\% fetal bovine serum (Millipore Sigma) under standard culture conditions (antibiotics, $37^{\circ} \mathrm{C}$ and $5 \% \mathrm{CO}_{2}$ in a humidified incubator). The study involved three experimental groups: Group I (Control), Group II (Ischemia only) (ISC), and Group III (Ischemia and reperfusion) (ISC/R). The SMCs of passage 2-4 were used for the study and the experiments were conducted in triplicate. The ischemia was simulated in vitro by treating the cells in ischemic buffer $\left(118 \mathrm{mM} \mathrm{NaCl}, 24 \mathrm{mM} \mathrm{Na}_{2} \mathrm{HCO}_{3}, 1 \mathrm{mM} \mathrm{Na}_{2} \mathrm{HPO}_{4}, 2.5 \mathrm{mM} \mathrm{CaCl}_{2}, 1.2 \mathrm{mM}, \mathrm{MgCl}_{2}, 20 \mathrm{mM}\right.$ sodium lactate, $16 \mathrm{mM} \mathrm{KCl}, 10 \mathrm{mM}$ 2-deoxyglucose and $\mathrm{pH}$ 6.2) for $15 \mathrm{~min}$ and reperfusion was attained by replacing the ischemic buffer with complete culture media overnight. The cells cultured and maintained in complete media served as control.

\section{Gene expression}

The CASMCs from the three experimental groups (Control, ISC, and ISC/R) were treated accordingly as mentioned above, total cellular RNA was isolated via the trizol method, cDNA was synthesized using $1 \mu \mathrm{g}$ RNA from each group using cDNA synthesis kit (Promega) following the manufacturer's protocol. The mRNA transcripts for the genes 5LPOX, 12LPOX, 15LPOX, COX2, ChemR23, GPCR18, and GPCR120 were amplified and quantified by real-time PCR (Applied Biosystems, CA, U.S.A.) using SYBR Green chemistry following our previous protocol [16]. The 18s rRNA was used as a housekeeping reference gene. The corresponding forward and reverse primers employed for each gene are displayed in Supplementary Table 1. The program for qRT-PCR was $95^{\circ} \mathrm{C}$ for $10 \mathrm{~min}$; and 40 cycles of $95^{\circ} \mathrm{C}$ for $15 \mathrm{sec}$, and $60^{\circ} \mathrm{C}$ for 1 min. The CT values for mRNA expression of the genes were normalized with the expression level of $18 \mathrm{~s}$ rRNA and the fold-change of mRNA expression was calculated by $2^{\wedge-\Delta \Delta C T}$ method. The experiments were conducted in triplicates and the results were presented as fold-change with respect to normal control [16].

\section{Immunofluorescence}

The CASMCs were maintained as mentioned above (Control, ISC, and ISC/R) in chamber slides, fixed with $10 \%$ formalin for 20 min and immunofluorescence of 5LPOX, 12LPOX, 15LPOX, COX2, ChemR23, GPCR18, and GPCR120 were performed [17]. The primary antibodies and fluorochrome-conjugated 
secondary antibodies were diluted 1:400 and 1:500, respectively. The experiments were done in triplicates as noted previously and the results were represented as $\log _{2}$ fold-change expression based on VRC.

\section{Capillary Western blot}

Capillary Western blot analyses were performed on a ProteinSimple Wes ${ }^{\circledR}$ System according to the manufacturer's instructions. Briefly, the samples were diluted to $0.5 \mu \mathrm{g} / \mu \mathrm{l}$ with $0.1 \mathrm{x}$ sample buffer followed by combining four parts of diluted sample with one part of $5 x$ fluorescent master mix and denatured at $95^{\circ} \mathrm{C}$ for $5 \mathrm{~min}$. Protein samples, blocking reagent, primary antibodies (GAPDH 1:100 and all others 1:20), HRP-conjugated secondary antibodies, and chemiluminescent substrate were loaded onto the cartridge and analysis was performed on 12-230 kDa Separation Module (Jess/Wes Separation Module, $8 \times 25$ capillary cartridges). The separation electrophoresis and immunodetection were done in the fully automated capillary system using the default program with stacking and separation at $475 \mathrm{~V}$ for 30 $\mathrm{min}$, blocking reagent for $5 \mathrm{~min}$, and both primary and secondary antibodies for $30 \mathrm{~min}$ followed by luminol/peroxide chemiluminescence detection for $15 \mathrm{~min}$. The experiments were conducted in duplicates and band area was calculated from the MFI using ImageJ software using 'Gels' mode, normalized to GAPDH and were represented as $\log _{2}$ fold-change expression based on VRC.

\section{RvE1 ELISA}

The cell culture supernatants of control, ISC, and ISC/R CASMCs were concentrated using (Amicon Ultra 15) and RvE1 level was detected by ELISA using the commercially available (MBS744335, MyBioSource) kit following the manufacturer's guidelines. Briefly, samples were allowed to bind to RvE1 antibody-coated wells followed by the incubation of Resolvin E1-HRP conjugate for 1 hour at $37^{\circ} \mathrm{C}$. The wells were washed carefully using the wash buffer provided with the kit and incubated with the HRP substrate under dark followed by termination of the reaction by adding stopping solution. The absorbance was read at $450 \mathrm{~nm}$ using a plate reader (Perkin Elmer) and the standard curve was employed to calculate the concentration of RvE1 (pg/ml) released from the CASMCs of the experimental groups [18].

\section{Statistical analysis}

The MFI values for swine $(n=3)$ tissues and cell culture experiments $(n=3)$ were expressed as mean \pm SEM. The nuclei were counted using ImageJ software with 'Analyze particle' mode and the MFI values were normalized to 100 cells. The average MFI/100 cells for each specimen/cell culture experimental group were calculated from the average MFI of 2-4 images randomly acquired from different microscopic fields. The statistical significance of the swine tissue specimen was calculated using One-Way ANOVA with Tukey's multiple comparison test using GraphPad prism software. However, due to the increased variation among swine groups and the limited sample size, statistical significance for the linear trend was considered for comparison. Hence, the $P<0.05$ for linear trend was considered significant in tissue 
specimen. The outlier images/specimen that displayed background fluorescence due to tissue folding were exempted from the analysis. The statistical analysis for qRT-PCR $(n=3)$, and ELISA $(n=2)$ were determined by One-Way ANOVA in a similar fashion. Additionally, the statistical analysis for western blot was determined using an unpaired t-test. The level of significance was set at $P<0.05$ for all experiments.

\section{Results}

\section{CABG and Harvest of LAD Artery}

The CABG was successfully performed in four Yucatan microswine and the animals recovered to normal cage activities within 7-10 days following the procedure. The animals were continued on a high-caloric diet until sacrifice and the intact $L A D$ ( $L A D-C A B G)$ adjacent to the anastomoses was harvested from each animal. Similarly, LAD arteries from atherosclerotic pigs (LAD-AS) and normal (LAD-C) pigs were harvested from identical anatomical locations. The harvested tissues were utilized for histomorphometry and immunostaining.

\section{Histology}

The histomorphometric analysis revealed considerable alterations in the tissue architecture in LAD-CABG and LAD-AS vessels when compared with the control (Figure 1). The H\&E, trichrome, and pentachrome staining revealed an intact medial layer in the control specimen (blue star); however, LAD-CABG and LADAS tissues displayed a more complex picture: intact medial layer towards adventitia and disorganized media towards intimal layer (blue star). The control tissues displayed an intact intimal layer (violet star), whereas the intimal layer was not well defined and intimal hyperplasia was evident in LAD-CABG and LAD-AS (black star). In addition, the trichrome staining revealed the deposition of collagen at the interface of diffused medial and intimal layers in LAD-CABG and LAD-AS tissues (yellow star), which suggests the ECM deposition by actively proliferating SMCs. Similarly, the pentachrome staining revealed increased collagen deposition in LAD-CABG when compared to LAD-AS as evident from intense yellow staining. Moreover, the mucin deposition (red star) was higher in LAD-AS and in control but proved negligible in LAD-CABG. In addition, the lipid rich necrotic core and fibrous cap were vividly evident in the histological sections of $L A D-C A B G$ and $L A D-A S$, suggesting the persistence and progression of atherosclerosis. Altogether, LAD-CABG and LAD-AS displayed similar histopathology.

\section{Protein expression of PLMs in swine tissues}

The protein expression of 5LPOX was significantly increased in LAD-CABG $(P=0.0025)$ and LAD-AS $(P=0.0048)$ compared to the control (Figures 2A and 5A); however, the increase was statistically not significant in $L A D-C A B G$ vs LAD-AS $(P=0.7597)$ (Figures $2 A$ and $5 A)$. Moreover, the linear trend of the increase was statistically significant $(P=0.0005)$. The protein expression of $12 \mathrm{LPOX}$ revealed a trend of decreased levels in LAD-CABG $(P=0.3155)$ and LAD-AS $(P=0.1881)$ compared to the control, but this trend 
was not statistically significant. In addition, the 12LPOX level in LAD-CABG was greater than LAD-AS, but this finding proved statistically insignificant $(P=0.9092)$ (Figures $2 B$ and $5 A)$. The protein level of 15LPOX was relatively decreased in $L A D-C A B G$ and $L A D-A S(P=0.7587, P=0.8191$, respectively) when compared with control; however, this finding was not statistically significant. Additionally, LAD-CABG and LAD-AS $(P=0.9930)$ exhibited a similar level of 15LPOX expression (Figures $3 A$ and $5 A$ ). The protein expression of GPCR18 was significantly decreased in LAD-CABG $(P=0.0319)$ and LAD-AS $(P=0.0012)$ compared to the control and significantly decreased in LAD-CABG compared to LAD-AS $(P=0.0342)$ (Figures 3B and 5A). The expression of COX2 was relatively higher in the control than LAD-CABG and LAD-AS and a decreased COX2 level was observed for LAD-AS than LAD-CABG; however, this finding was not statistically significant ( $P=0.4135, P=0.1095, P=0.5606$, respectively for control vs $L A D-C A B G$, control vs $L A D-A S$, LADCABG and LAD-AS) (Figures 4A and 5A). However, there was a linear trend for decreased COX2 expression $(P=0.0501)$. The level of ChemR23 receptor was relatively lower in LAD-CABG and LAD-AS than the control, whereas there was no considerable difference between LAD-CABG and LAD-AS, but the results were not statistically significant $(P=0.9172, P=0.3292, P=0.5128$, respectively for control vs $L A D-$ CABG, control vs LAD-AS, LAD-CABG and LAD-AS) (Figures 4A and 5A). The protein expression of GPCR120 was decreased in LAD-AS when compared with control, whereas the level of GPCR120 was decreased in $\angle A D-C A B G$ vs control; however, the differences were not statistically significant ( $P=0.9357$ and $P=0.0801$, respectively for control vs $L A D-C A B G$, and control vs LAD-AS). GPCR 120 was significantly increased in LAD-CABG compared with LAD-AS $(P=0.0518)$ and the linear trend for GPCR120 alteration was statistically significant $(P=0.0360)$ (Figures 4B and 5A).

\section{Protein expression of PLMs in cultured CASMCs}

The protein expression of 5LPOX in CASMCs was significantly increased in ISC group $(P<0.0001)$ and ISC/R group $(P=0.0508)$ compared with control and significantly decreased in ISC/R group $(P=0.0004)$ than ISC group. The protein level of 12LPOX was significantly increased in ISC ( $P=0.0357)$ and nonsignificantly in ISC/R $(P=0.2009)$ groups when compared with the control and was decreased in ISC/R compared with ISC group; however, the decrease was not statistically significant $(P=0.4087)$. The level of 15LPOX was significantly increased in ISC $(P<0.0001)$ and ISC/R $(P=0.0002)$ group when compared with the control and was significantly decreased in ISC/R than ISC group $(P=0.0003)$. The protein level of COX2 was increased in ISC $(P=0.1047)$ and ISC/R $(P=0.0095)$ group when compared with the control; however, the increase in ISC group was statistically not significant. Notably, COX2 was lower in ISC group compared with ISC/R group; however, the decrease was statistically not significant $(P=0.1822)$ in ISC/R when compared with ISC group. The expression of ChemR23 was significantly higher in ISC/R group when compared to control $(P=0.0009)$ and ISC groups $(P=0.0080)$, while ChemR23 level was significantly higher in ISC than control groups $(P=0.0333)$. ISC $(P=0.7571)$ and ISC/R $(P=0.7259)$ groups exhibited a slight increase and decrease in GPCR18 respectively with respect to the control, but the difference between ISC group and ISC/R groups $(P=0.3507)$ was not significant. The protein expression of GPCR120 was significantly higher in ISC $(P=0.0072)$ and ISC/R groups $(P=0.0441)$ compared with the control. ISC displayed increased GPCR120 compared with ISC/R; however, the increase was not statistically 
significant $(P=0.3069)$. The representative images are displayed in Figure 5B and the respective bar diagrams with statistical analysis are given in Figure 5C.

\section{mRNA transcription}

mRNA transcripts for all the genes, 5LPOX, 12LPOX, 15LPOX, COX2, ChemR23, GPCR18, and GPCR120 were upregulated in ISC groups when compared with the control SMCs; however, the increase was not statistically significant with the notable exceptions of 12LPOX $(P=0.0190)$ and 15LPOX ( $P=0.0531)$. The mRNA levels of 5LPOX, 12LPOX, 15LPOX, ChemR23, and GPCR18 were downregulated considerably in ISC/R group than the ISC group; however, this proved statistically insignificant. In addition, similar levels of 12LPOX, 15LPOX, and ChemR23 expression, upregulation of 5LPOX, and downregulation of GPCR18 was observed in reperfused CASMCs relative to the control. Moreover, COX2 expression was significantly higher in ISC/R group when compared with the control and while a similar trend was observed for GPCR120 this was statistically insignificant. The fold-change expression relative to the housekeeping gene 18s rRNA is displayed in Figure 5D.

\section{Western Blot}

Western blot analysis revealed the upregulation of 5LPOX and ChemR23 in ISC group when compared with the control, whereas the reperfusion resulted in further upregulation. However, apart from ChemR23 in ISC/R group ( $P=0.0008$ ), the increase was not statistically significant. GPCR120 and 15LPOX were downregulated in both ISC and ISR/R groups compared with the control, whereas the downregulation was significant for 12LPOX in ISC group ( $P=0.0192)$. COX2 was significantly downregulated in ISC group $(P<0.0001)$ and non-significant upregulation was exhibited in ISC/R group with respect to the control. GPCR18 was significantly downregulated in ISC/R groups $(P=0.0453)$ and upregulated in ISC group compared with the control; however, the increased expression was not statistically significant. All biomarkers except GPCR18 were upregulated upon reperfusion; however, the difference was statistically not significant. The results were normalized to the expression of the house keeping gene, GAPDH (Figures 6E and 6F).

\section{RvE1 ELISA}

The level of RvE1 was significantly higher $(128.61 \%$ increase, $P=0.0065)$ in the cell culture supernatant of ISC CASMCs and upon reperfusion significantly declined (123.1\% decrease, $P=0.0422)$ when compared with the control. In addition, the RvE1 in ISC/R group was significantly lower (158.3\% decrease, $P=0.0005$ ) when compared with ISC CASMCs. Overall, the ISC group displayed an increased RvE1 level and ISC/R group exhibited a decreased level (Figure 6G).

\section{Discussion}


An astounding 400,000 CABG surgeries are annually performed in the United States. The persistent inflammation and ischemia impede regenerative responses in post-CABG cardiac performance [19], [20]. Similarly, surviving coronary arteries adjacent to anastomoses exhibit a similar inflammatory status and sustained ischemic insults due to the pro-atherogenic events, including SMC hyperplasia [10].

Nevertheless, autologous vein grafts have been hailed for the durability that ensures revascularization to the infarcted myocardium and a large body of evidence is available regarding effective strategies to prevent vein graft failure. Interestingly, a resolution of inflammation via the activation of PLM attenuating the hyperplasia subsequently accelerates the healing response in the vein graft [21]. However, information regarding the resolution of inflammation in coronary artery tissue adjacent to the anastomosis site remains limited. A persistent atherosclerotic plaque and/or coronary occlusion hinders the ability of the surviving coronary artery to withstand post-CABG ischemic insults and the subsequent resolution of inflammatory events greatly influence the performance of the vein graft. Hence, we investigated the status of PLM mediators in the LAD adjacent to the anastomosis site in swine CABG model and to understand the expression of these mediators in response to ischemia and reperfusion in cultured CASMCs.

The current study utilized our established swine CABG model created by anastomosing the left internal mammary artery (LIMA) to LAD using superficial epigastric vein graft [10]. The histological sections of LAD-CABG and LAD-AS revealed a growing necrotic core and intimal hyperplasia, the classical features of atherosclerosis as reported elsewhere [22] [23]. Interestingly, a recent seminal study reported that LAD adjacent to anastomoses to be the most affected artery in human IHD with the clinical and histological presentation of severe atherosclerosis [24]. Intimal hyperplasia is the consequence of SMC activation and phenotype switch resulting in the increased deposition of ECM [25]. In addition, the increased foam cell formation propagates inflammation resulting in the formation and maturation of a necrotic core and subsequent proliferation of SMCs [26]. Moreover, the hypersecretion and hyperactivity of various MMPs result in increased digestion of ECM components, which in turn induces ECM disorganization [27]. Overall, the pro-atherogenic signals and subsequent vascular ischemia substantially increase the proinflammatory pool in the post-CABG coronary artery. Therefore, it is logical that the surviving vascular tissue respond and/or adapt to withstand such ischemic/inflammatory insults by secreting PLMs.

Previous studied revealed the ability of mammalian systems to synthesize PLMs from $\omega-3$ PUFAs to elicit potent anti-inflammatory effects to resolve inflammation in diverse tissue types. The PUFAs including EPA and DHA give rise to the PLMs, especially resolvin E (RvE) series and RvD series, respectively [28]. The major lipoxygenase enzymes, including COX2, 5LPOX, 12LPOX, and 15LPOX, metabolize EPA and DHA and result in the formation of RvE and RvD, which in turn elicit an immunomodulatory function via GPCR that facilitates the expression of multiple mediators associated with the resolution of inflammation including enzymes, chemokines, cytokines, and growth factors [29]. Interestingly, the immunomodulatory effects of PLMs do not compromise the host immune system to promote tissue resolution. For instance, the RvD1 interfere with the proinflammatory function of leukotriene B4 (LTB4) via an unknown mechanism. In addition, RVD1 lowers 5LPOX activity and LTB4 synthesis, while switching the signaling flux favoring the synthesis of RvD [30]. 
Four PLM receptors have been characterized and investigated for their therapeutic opportunities to settle inflammation in several chronic diseases [1]. However, little is known regarding PLM signaling in IHD, especially in post-CABG coronary complications. GPCR32, GPCR18, and ChemR23 recognize RvD1, RvD2, and RvE1 respectively and these receptors were reported to be expressed in immune as well as nonimmune cells [1]. In addition, GPCR120 binds to $\omega$-3 PUFAs, which in turn are the major precursors to PLMs [31]. Therefore, the level of LPOX (5LPOX, 12LPOX and 15LPOX) mediating PLM synthesis and the expression status of PLM receptors provide better insight into the extent of pro-resolvin signaling in inflammatory tissues. Interestingly, PLM signaling has been recognized in the resolution of sterile inflammatory events associated with ischemia/reperfusion injury, alluding to an involvement in IHD [1]. Thus, it is reasonable to speculate that the level of these mediators reflects the inflammatory status of the post-CABG coronary artery.

Our findings displayed the upregulation of PLM synthesis enzymes 5LPOX in the LAD-CABG tissues compared with the normal control. On the other hand, other mediators, including GPCR18, were relatively decreased in LAD-CABG compared with the control. Notably, the ischemic CASMCs displayed an increased trend of upregulating all the LPOX enzymes and receptors with respect to control at both transcript and protein levels. The increased level of ChemR23 following the reperfusion suggests the resolution of inflammation triggered by the ischemia-driven signals and warrants further research. In addition, the expression status of 5LPOX and ChemR23 at protein level were relatively higher in ischemic groups as confirmed by western blot. Similarly, 12LPOX, 15LPOX, COX2, and GPCR120 were lower than the control relative to the house keeping gene expression. Together, 5LPOX and ChemR23 displayed upregulation at protein and transcript levels in ischemic SMCs and a similar increase was observed at protein level in LAD-CABG tissue sections. These findings suggest that 5LPOX and ChemR23 play a crucial role in the resolution of vascular inflammation in the coronary arteries contending with a pathologic episode of persistent ischemia.

The PUFA-5LPOX-ChemR23 axis has been associated with the synthesis pathway of RvE1, where RvE1 directly activates ChemR23 in mononuclear cells and inhibits the proinflammatory LTB4 receptor in neutrophils. Moreover, the ChemR23-RvE1 axis alleviates the TNF-stimulated activation of NF-KB attenuating the inflammatory responses [32]. In addition, RvE1 prevents the transendothelial migration of immune cells and reduces the release of superoxide generation and subsequent oxidative stress in the vascular system. Furthermore, the ChemR23-RvE1 axis significantly reduced the IL-12 secretion promoting the resolution of inflammation as reported elsewhere [33]. However, limited information is available on the expression status of RvE1 in ischemic CASMCs; expanding our knowledge of the expression status of RvE1 in ischemic CASMCs possesses immense translational potential, as SMCs play crucial role in coronary atherosclerosis and post-CABG ischemic insults. The increased levels of 5LPOX and ChemR23 in the LAD-CABG and cultured SMCs, along with the information regarding their involvement in RvE1 synthesis, led us to determine secreted RvE1 in the SMC culture. Interestingly, our finding revealed a significantly higher level of RvE1 in ischemic SMCs than the control, which considerably decreased following reperfusion suggesting the possible role of RvE1 in anti-inflammatory responses for the resolution of coronary pathology in SMC. Additionally, the decreased level of ChemR23 
in ischemic SMCs compared with the reperfused cells suggests that a potentially insufficient level of RvE1 and/or the ChemR23-inhibitory effects of ischemia. However, extensive investigations are warranted to unveil the mechanism of RvE1 induction and ChemR23-inhibitory effects of ischemia and downstream cytokine signaling in post-CABG-LAD. Overall, our findings suggest that the SMCs in the coronary vasculature withstand the ischemia-triggered proinflammatory episodes by increasing the secretion of RvE1.

Overall, in this pilot study we successfully established the involvement of RvE1 and several PLM mediators in post-CABG coronary survival. The study simulated human atherosclerosis, CABG procedures, and post-CABG survival in a swine model and established the expression status of PLM mediators. Importantly, this is the first study investigating the association of PLM mediators and receptors in post-CABG coronary pathology. However, the study warrants further investigation to unveil the molecular signaling underlying the regulation of the expression of PLMs in vascular tissue, which would reveal multiple translational avenues for the management of IHD. The major limitations of the study include: (i) lesser sample population and associated variability, (ii) unavailability of swine specific antibodies for protein detection experiments, (iii) the relative smaller size of LAD which prevented transcriptional and western blot analyses to examine the protein expression in the tissue, (iv) the normal control used for comparison was miniswine assuming similar biochemistry and molecular pathology, and (v) the study focused on RvE1 signaling and the members of E and D series resolvins, and other PLMs, particularly maresins and protectins, warrant further attention. Nevertheless, the findings firmly established a pathway for the resolution of inflammation mediated by 5LPOX-ChemR23-RvE1 axis.

\section{Conclusions}

RvE1 synthetic enzyme 5LPOX was increased in LAD-CABG tissues and ischemic CASMCs, whereas ChemR23 and RvE1 receptor were decreased in LAD-CABG and increased in ischemic CASMCs with a concomitant upregulation following the reperfusion. The level of RvE1 was increased in ischemic cells and declined following the reperfusion, suggesting a possible role of ischemia to increase the level of ChemR23 and facilitate the RvE1-mediated resolution of inflammation. A more sophisticated understanding of the underlying mechanisms of RvE1 induction by SMCs under ischemia insults warrants further investigation, which would open novel translational avenues to improve post-CABG coronary survival.

\section{Declarations}

Compliance with Ethical Standards

\section{Funding:}

This study was funded by research grants R01 HL128063, R01 HL144125, and R01 HL147662 to DKA from the National Institutes of Health, USA. The content of this article is solely the responsibility of the 
authors and does not necessarily represent the official views of the National Institutes of Health.

\section{Consent to publish:}

As the corresponding author, I verify that all authors significantly contributed to various aspect of the study, have read the manuscript, and consented to submit for publication in Molecular Biology Reports.

\section{Conflict of interest:}

Author A has received startup research funds from Western University of Health Sciences. Author A declares that he has no conflict of interest. Author B declares that she has no conflict of interest. Author $\mathrm{C}$ declares that he has no conflict of interest. Author $\mathrm{D}$ declares that he has no conflict of interest. Author E declares that he has no conflict of interest.

\section{Ethical Approval:}

The animal studies were conducted with prior approval of the research protocol by the IACUC of Creighton University, Omaha, Nebraska, and the IACUC of Western University of Health Sciences, Pomona, California.

\section{Consent to participate:}

This article does not contain any studies with human participants performed by any of the authors.

\section{References}

1. Pirault J, Bäck M (2018) Lipoxin and Resolvin Receptors Transducing the Resolution of Inflammation in Cardiovascular Disease. Front Pharmacol 9. https://doi.org/10.3389/fphar.2018.01273

2. Kain V, Prabhu SD, Halade GV (2014) Inflammation revisited: inflammation versus resolution of inflammation following myocardial infarction. Basic Res Cardiol 109:444. https://doi.org/10.1007/s00395-014-0444-7

3. Conte MS, Desai TA, Wu B et al Pro-resolving lipid mediators in vascular disease.J Clin Invest128:3727-3735. https://doi.org/10.1172/JCl97947

4. Motwani MP, Colas RA, George MJ et al Pro-resolving mediators promote resolution in a human skin model of UV-killed Escherichia coli-driven acute inflammation.JCI Insight3:. https://doi.org/10.1172/jci.insight.94463

5. Dalli J, Serhan CN (2017) Pro-Resolving Mediators in Regulating and Conferring Macrophage Function. Front Immunol 8:1400. https://doi.org/10.3389/fimmu.2017.01400 
6. Sansbury Brian E, Spite Matthew (2016) Resolution of Acute Inflammation and the Role of Resolvins in Immunity, Thrombosis, and Vascular Biology. Circ Res 119:113-130.

https://doi.org/10.1161/CIRCRESAHA.116.307308

7. Barden AE, Mas E, Croft KD et al (2015) Specialized proresolving lipid mediators in humans with the metabolic syndrome after $n-3$ fatty acids and aspirin. Am J Clin Nutr 102:1357-1364. https://doi.org/10.3945/ajcn.115.116384

8. Kain V, Ingle KA, Colas RA et al (2015) Resolvin D1 activates the inflammation resolving response at splenic and ventricular site following myocardial infarction leading to improved ventricular function. J Mol Cell Cardiol 84:24-35. https://doi.org/10.1016/j.yjmcc.2015.04.003

9. Liu G, Liu Q, Shen Y et al (2018) Early treatment with Resolvin E1 facilitates myocardial recovery from ischaemia in mice. Br J Pharmacol 175:1205-1216. https://doi.org/10.1111/bph.14041

10. Thankam FG, Ayoub JG, Ahmed MMR et al (2020) Association of hypoxia and mitochondrial damage associated molecular patterns in the pathogenesis of vein graft failure: A PILOT STUDY. Transl Res. https://doi.org/10.1016/j.trsl.2020.08.010

11. Bulow NMH, Colpo E, Duarte MF et al (2014) Inflammatory Response in Patients under Coronary Artery Bypass Grafting Surgery and Clinical Implications: A Review of the Relevance of Dexmedetomidine Use. In: Int. Sch. Res. Not. https://www.hindawi.com/journals/isrn/2014/905238/. Accessed 3 Nov 2019

12. Thankam FG, Dilisio MF, Dietz NE, Agrawal DK (2016) TREM-1, HMGB1 and RAGE in the Shoulder Tendon: Dual Mechanisms for Inflammation Based on the Coincidence of Glenohumeral Arthritis. PLoS ONE 11:e0165492. https://doi.org/10.1371/journal.pone.0165492

13. Thankam FG, Roesch ZK, Dilisio MF et al (2018) Association of Inflammatory Responses and ECM Disorganization with HMGB1 Upregulation and NLRP3 Inflammasome Activation in the Injured Rotator Cuff Tendon. Sci Rep 8:1-14. https://doi.org/10.1038/s41598-018-27250-2

14. Thankam FG, Boosani CS, Dilisio MF et al (2016) MicroRNAs Associated with Shoulder Tendon Matrisome Disorganization in Glenohumeral Arthritis. PLoS ONE 11:e0168077. https://doi.org/10.1371/journal.pone.0168077

15. Dhume AS, Agrawal DK (2003) Inability of vascular smooth muscle cells to proceed beyond S phase of cell cycle, and increased apoptosis in symptomatic carotid artery disease 1 1Competition of interest: none. J Vasc Surg 38:155-161. https://doi.org/10.1016/S0741-5214(02)75463-3

16. Thankam FG, Chandra IS, Kovilam AN et al (2018) Amplification of Mitochondrial Activity in the Healing Response Following Rotator Cuff Tendon Injury. Sci Rep 8. https://doi.org/10.1038/s41598018-35391-7

17. Thankam FG, Chandra IS, Kovilam AN et al (2018) Amplification of Mitochondrial Activity in the Healing Response Following Rotator Cuff Tendon Injury. Sci Rep 8:1-14. https://doi.org/10.1038/s41598-018-35391-7

18. Günay S, Taşova F, Yılmaz HE et al (2019) Serum Resolvin E1 Levels and Its Relationship with Disease Activity in Ulcerative Colitis. In: Gastroenterol. Res. Pract. 
https://www.hindawi.com/journals/grp/2019/6258327/. Accessed 15 Oct 2020

19. Alam SR, Stirrat C, Spath N et al (2017) Myocardial inflammation, injury and infarction during onpump coronary artery bypass graft surgery. J Cardiothorac Surg 12. https://doi.org/10.1186/s13019017-0681-6

20. Warltier DC, Laffey JG, Boylan JF, Cheng DCH (2002) The Systemic Inflammatory Response to Cardiac Surgerylmplications for the Anesthesiologist. Anesthesiology 97:215-252. https://doi.org/10.1097/00000542-200207000-00030

21. Wu B, Werlin EC, Chen M et al (2018) Perivascular delivery of resolvin D1 inhibits neointimal hyperplasia in a rabbit vein graft model. J Vasc Surg 68. https://doi.org/10.1016/j.jvs.2018.05.206. :188S-200S.e4

22. Insull W (2009) The Pathology of Atherosclerosis: Plaque Development and Plaque Responses to Medical Treatment. Am J Med 122:S3-S14. https://doi.org/10.1016/j.amjmed.2008.10.013

23. van der Wal AC (2007) Coronary artery pathology. Heart 93:1484-1489. https://doi.org/10.1136/hrt.2004.038364

24. Pérez Sorí Y, Herrera Moya VA, Puig Reyes I et al (2019) Histología de la placa de ateroma en arterias coronarias de fallecidos después de una revascularización miocárdica quirúrgica. Clínica E Investig En Arterioscler 31:63-72. https://doi.org/10.1016/j.arteri.2018.07.002

25. Wadey K, Lopes J, Bendeck M, George S (2018) Role of smooth muscle cells in coronary artery bypass grafting failure. Cardiovasc Res 114:601-610. https://doi.org/10.1093/cvr/cvy021

26. Lim S, Park S (2014) Role of vascular smooth muscle cell in the inflammation of atherosclerosis. BMB Rep 47:1-7. https://doi.org/10.5483/BMBRep.2014.47.1.285

27. Hu D, Yin C, Luo S et al (2019) Vascular Smooth Muscle Cells Contribute to Atherosclerosis Immunity. Front Immunol 10. https://doi.org/10.3389/fimmu.2019.01101

28. Leigh NJ, Nelson JW, Mellas RE et al (2014) Expression of Resolvin D1 Biosynthetic Pathways in Salivary Epithelium. J Dent Res 93:300-305. https://doi.org/10.1177/0022034513519108

29. Serhan CN, Petasis NA (2011) Resolvins and Protectins in Inflammation-Resolution. Chem Rev 111:5922-5943. https://doi.org/10.1021/cr100396c

30. Fredman G, Ozcan L, Spolitu S et al (2014) Resolvin D1 limits 5-lipoxygenase nuclear localization and leukotriene B4 synthesis by inhibiting a calcium-activated kinase pathway. Proc Natl Acad Sci 111:14530-14535. https://doi.org/10.1073/pnas.1410851111

31. Oh DY, Talukdar S, Bae EJ et al (2010) GPR120 is an Omega-3 Fatty Acid Receptor Mediating Potent Anti-Inflammatory and Insulin Sensitizing Effects. Cell 142:687-698. https://doi.org/10.1016/j.cell.2010.07.041

32. Serhan CN, Chiang N, Van Dyke TE (2008) Resolving inflammation: dual anti-inflammatory and proresolution lipid mediators. Nat Rev Immunol 8:349-361. https://doi.org/10.1038/nri2294

33. Arita M, Ohira T, Sun Y-P et al (2007) Resolvin E1 Selectively Interacts with Leukotriene B4 Receptor BLT1 and ChemR23 to Regulate Inflammation. J Immunol 178:3912-3917. 


\section{Figures}

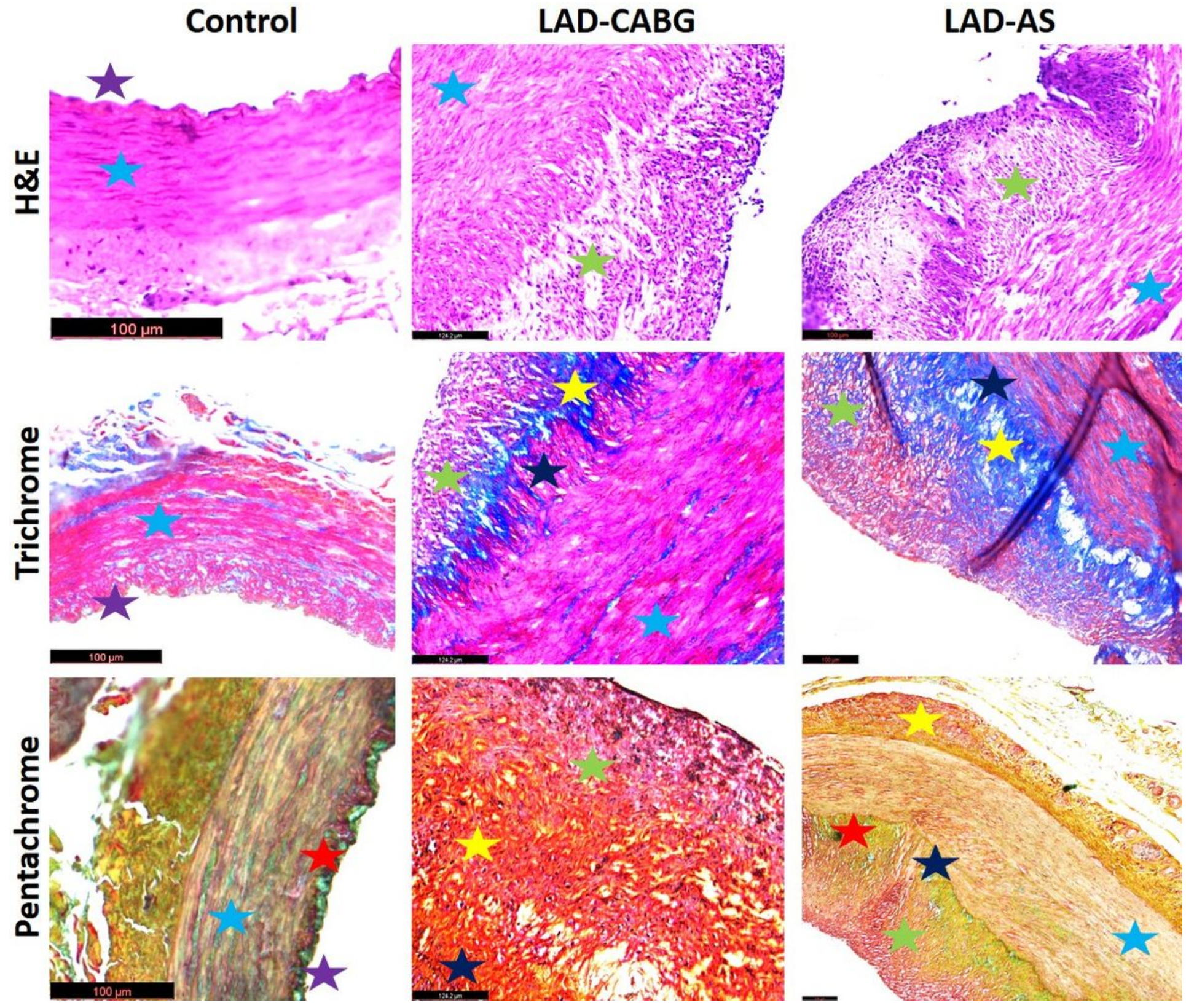

Figure 1

The histomorphology evaluations H\&E, Trichrome and Pentachrome staining: The evaluations were performed in four vessels LAD-Control, LAD-CABG, and LAD-AS. The blue star signifies medial layer orientation, violet star shows intact intimal layer, black star reveals intimal hyperplasia, yellow star, and yellow staining in pentachrome point collagen deposition, and the red star displays mucin deposition. The images were acquired in $20 x$ magnification. 

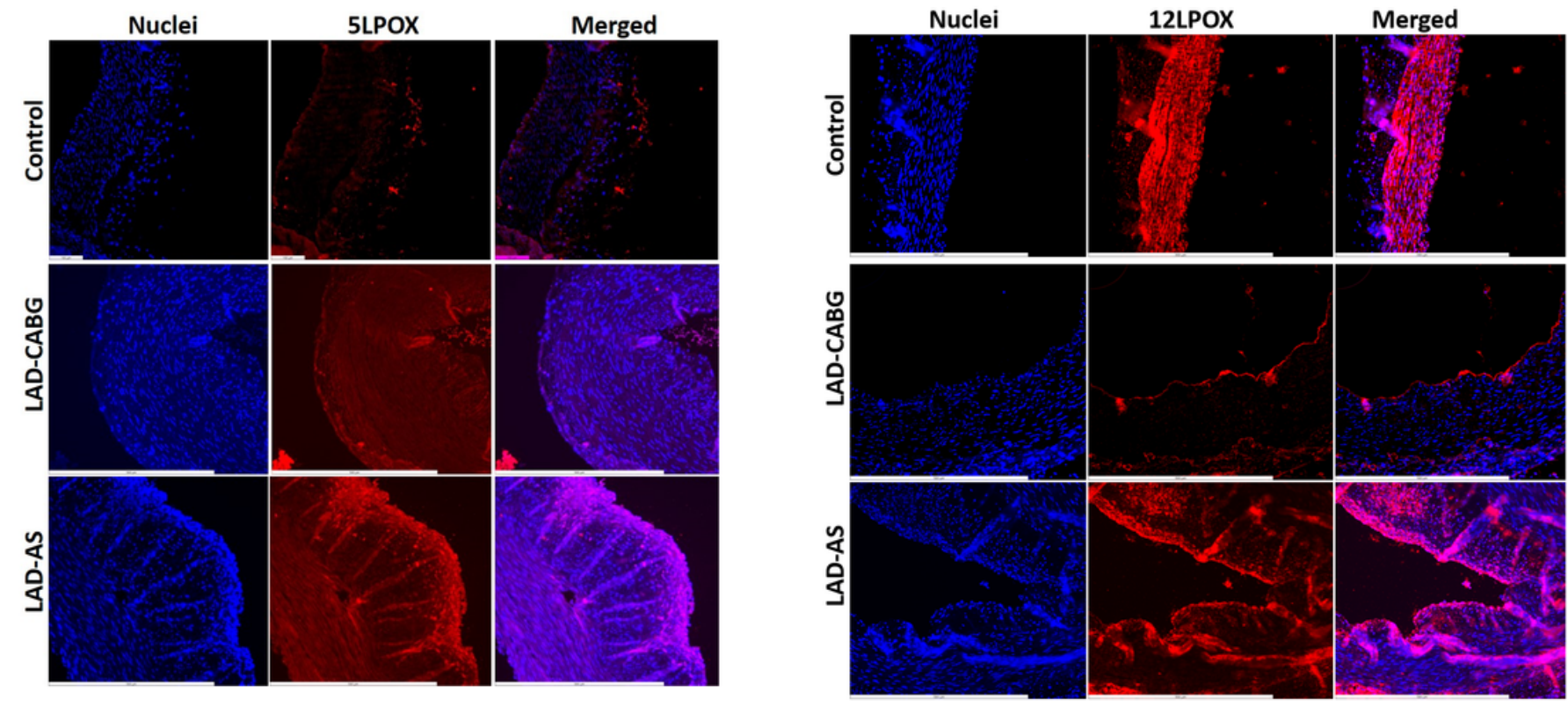

Figure 2

Representative images for the immunofluorescence analysis for the expression of (A) 5LPOX and (B) $12 \mathrm{LPOX}$ showing the altered expression in the LAD vessels. Images in the top row are histological sections of Control (LAD-C), followed by LAD-CABG (second row) and LAD-AS (third row). Images in the left column show nuclear staining with DAPl; the images in the middle column show expression of 5LPOX/12LPOX while the images in the right column show overlay of 5LPOX/12LPOX staining with DAPI. Images were acquired at 20x magnification.
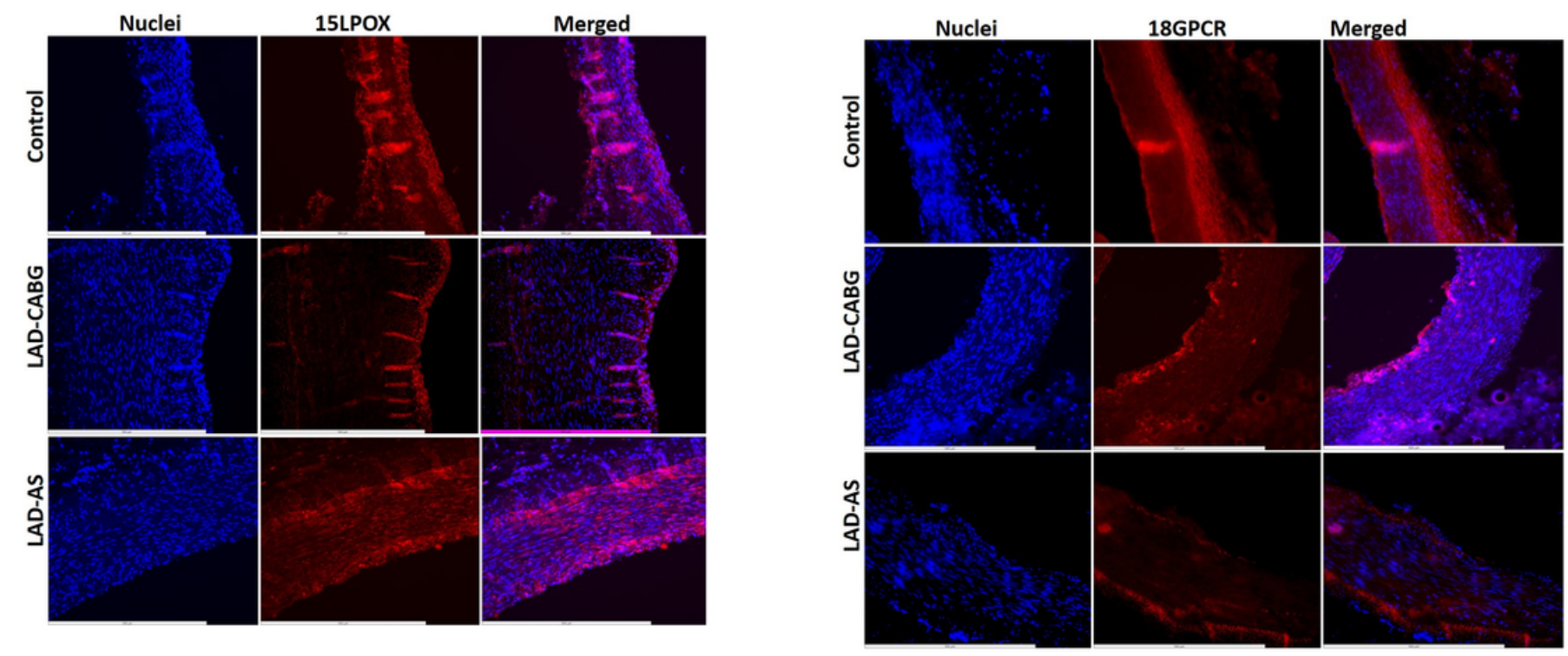

Figure 3 
Representative images for the immunofluorescence analysis for the expression of (A) 15LPOX and (B) GPCR18 showing the altered expression in the LAD vessels. Images in the top row are histological sections of Control (LAD-C), followed by LAD-CABG (second row) and LAD-AS (third row). Images in the left column show nuclear staining with DAPl; the images in the middle column show expression of 15LPOX/ GPCR18 while the images in the right column show overlay of 15LPOX/ GPCR18 staining with DAPI. Images were acquired at 20x magnification.
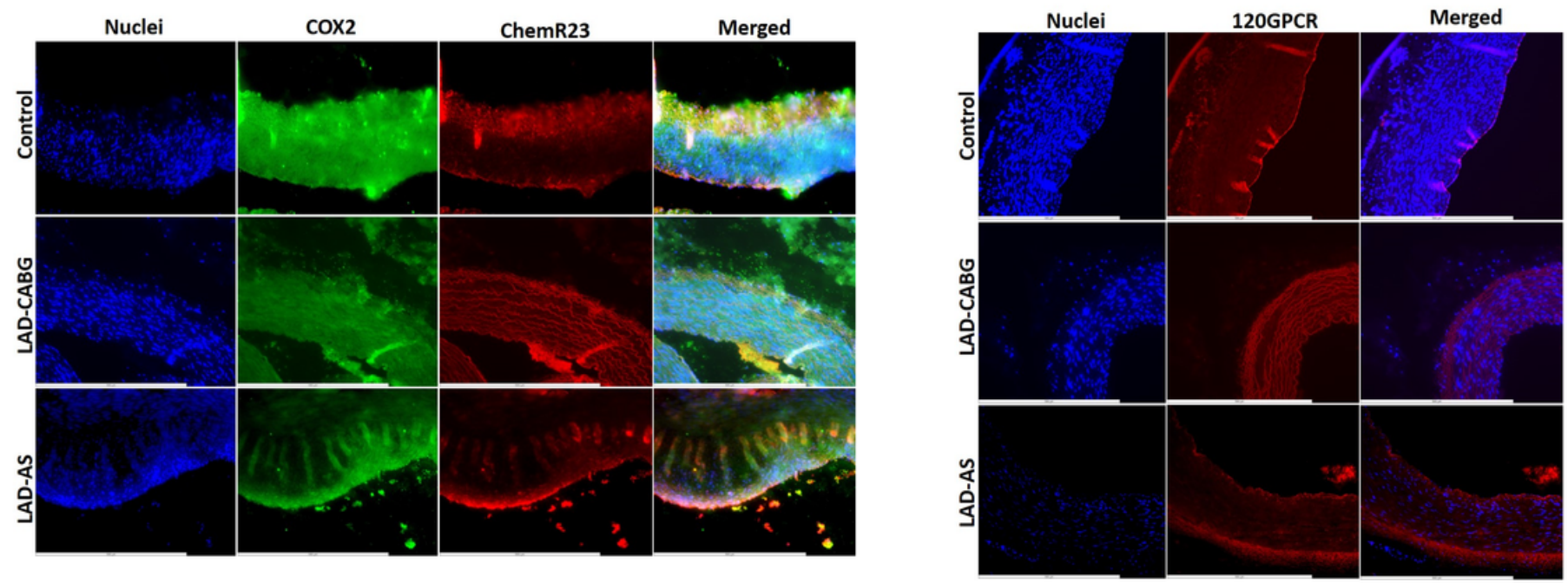

\section{Figure 4}

Representative images for the immunofluorescence analysis for the expression of (A) COX2 and ChemR23 and (B) GPCR120 showing the altered expression in the LAD vessels. Images in the top row are histological sections of Control (LAD-C), followed by LAD-CABG (second row) and LAD-AS (third row). Images in the left column show nuclear staining with DAPI; the images in the middle column show expression of COX2 and ChemR23 while the images in the right column show overlay of COX2 and ChemR23/ GPCR120 staining with DAPI. Images were acquired at 20x magnification. 

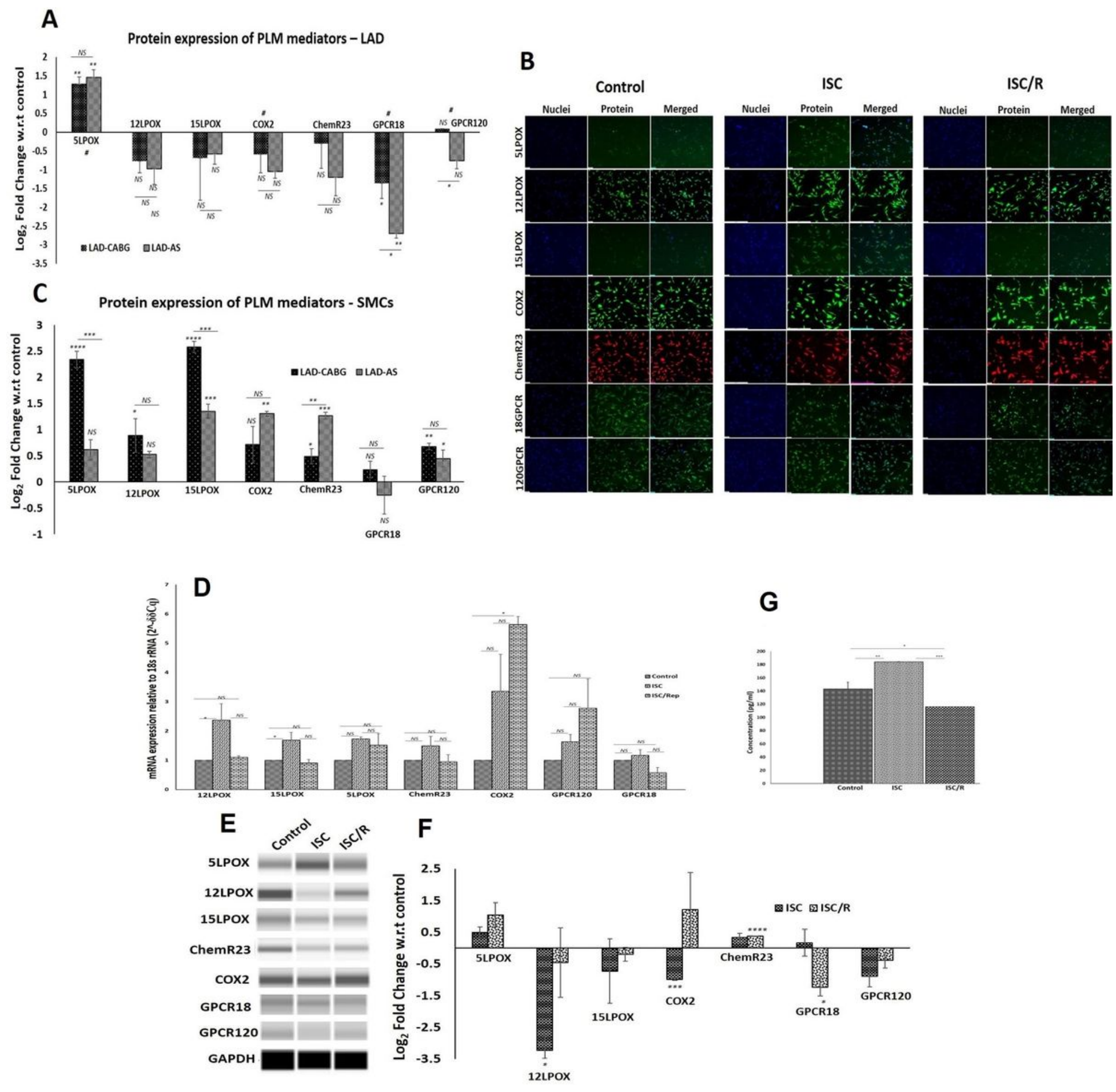

\section{Figure 5}

(A) The quantification of protein expression. The mean fluorescence intensity quantified from each specimen was normalized to 100 cells (MFI/100 cells) which was employed to calculate fold change based on the variation with respect to control (VRC). The graphs represent fold change values with standard error. The statistical significance based on One-Way ANOVA is represented in the figure. (B) Immunofluorescence analysis for the protein expression of the mediators of resolvin synthetic pathway by SMCs cultured under normal, ISC, ISC/R conditions: Images in the first column in each panel show nuclear staining with DAPI; the images in the middle column show the biomarker expression and the third 
column presents the merged images of the biomarkers with DAPI. Images were acquired at 20x magnification using CCD camera attached to the Leica Thunder microscope. (C) The image shows quantification, and the graphs represent the values of mean fold change with standard error. (D) qRT-PCR analysis for the mRNA expression of 5LPOX, 12LPOX, 15LPOX, COX2, ChemR23, GPCR18 and GPCR120 on SMCs cultured under ISC and ISC/R with respect to control. The results were expressed as fold change of expression relative to control. (E) Western Blot analysis for the protein expression of 5LPOX, 12LPOX, 15LPOX, COX2, ChemR23, GPCR18 and GPCR120 revealing the bands corresponding to the expression of proteins; lane 1 control, lane 2 ISC and lane 3 ISC/R. (F) Quantification of expression represented by fold change based on the housekeeping gene GAPDH. (G) Quantification of RvE1 level from the cell culture supernatant of CASMCs cultured under ISC, ISC/R compared to the control. The statistical significance is

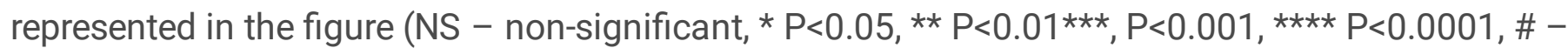
significance for the test for linear trend, $P<0.05)$.

\section{Supplementary Files}

This is a list of supplementary files associated with this preprint. Click to download.

- Supptable1.docx 